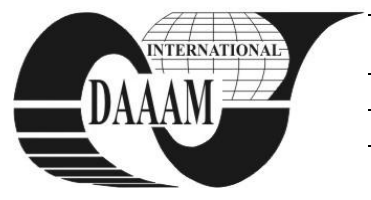

\title{
CONCEPTUAL MODELING OF THE SERIAL LOGISTIC SYSTEM
}

\author{
BUCKI, R[obert] \& CHRAMCOV, B[ronislav]
}

\begin{abstract}
The paper highlights the problem of mathematical modelling of a logistic system in which production stands are arranged in a series. The problem itself consists in determining the sequence of elements of the order vector which are to be realized subsequently. In order to avoid bringing the production process to a standstill each production stand can be multiplied on demand.The proposed heuristic algorithm of the relative order chooses the required element on which certain operations are carried out. The state of orders decreases after each production decision which influences the state of the whole logistic system at every stage. The lost flow capacity criterion is used on condition that it is associated by bounds.

Key words: mathematical modelling, heuristic algorithm, manufacturing criterion, logistic system
\end{abstract}

\section{INTRODUCTION}

In today's highly competitive world, there is an increasing pressure for companies to concentrate on continuous improvements. In the manufacturing environment this means that companies will often review their factory layouts or the way in which the factory is controlled and managed. Simulation of this systems can lead to cost minimizing procedures implemented in companies (Chramcov et al., 2011). While managing the system it is important to provide the correct information about the product at the accurate point of time (Bucki \& Chramcov, 2011). Knowing that there is an order to be realized, we can easily find out in advance what kind of charge will be necessary and what amount of it will be needed (Schmidt, 1988). In multi stage job problems, simple priority dispatching rules such as shortest processing time (SPT) and earliest due date (EDD) can be used to obtain solutions of minimum total processing time, but may not sometimes give sequences as expected that are close to optimal (Modrak \& Pandian, 2010). Case studies prove that production can be optimized by means of transformation production procedures (Modrak, 2009). Moreover, many problems can be solved by means of adequate multi-criteria decision-making using modern heuristics (Michalewicz \& Fogel, 2004).

\section{PROBLEM FORMULATION}

Let us assume that the sample logistic system consists of $I$ active logistic blocks arranged in a series. Each logistic block is meant to carry out a production operation. There are no buffer stores placed between logistic blocks in the discussed case. Each logistic block consists of a certain number of production stands. If a production stand in the logistic block does not have enough production capacity to accept all production flow, another parallel stand carrying out the same production operation is activated in this exact logistic block. If a production stand is not needed any more, it is deactivated in the discussed logistic block.

Let us introduce the vector of charges $W=\left[w_{l}\right], l=1, \ldots, L$ and the vector of orders $Z=\left[z_{n}\right], n=1, \ldots, N$ (given in units).
The assignment matrix of ordered products to charges takes the form $\Omega=\left[\omega_{n, l}\right]$. Let us introduce the vector of production machines $M_{i}=\left[m_{i, j}\right], i=1, \ldots, I, j=1, \ldots, J$, which carry out autonomous operations on the specified $n$-th product. The operations are realized subsequently.

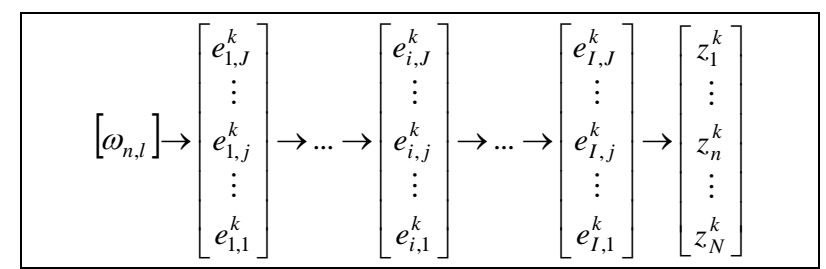

Fig. 1. The structure scheme of the serial logistic system

If the $n$-th product is realized by the $j$-th production stand at the $k$-th stage, $k=1, \ldots, K$, then $e_{i, j}^{k}=1$. Otherwise, $e_{i, j}^{k}=0$. Moreover, we assume that $\forall e_{i, 1}^{k}=1$. The structure of this serial logistic system is present in the Fig. 1.

Let $G=\left[g_{n, i}\right]$ be the life vector for a new brand set of tools for manufacturing the $n$-th product. At the same time $g_{n, i}=\psi$ if the $n$-th product is realized in the $j$-th production stand of the $i$-th logistic block, $\psi=1, \ldots, \Psi$, otherwise, $g_{n, i}=0$. We can assume that $\underset{1 \leq i \leq I}{\forall} g_{n, i}=\psi$. If the number $\psi$ is reached for the given $n$-th element of the vector $Z$ in the $j$-th production stand of the $i$-th logistic block, the tool in this exact stand has to be replaced by a new one.

Let $S_{n}^{k-1}=\left[s(n)_{i, j}^{k-1}\right]$ be the matrix of state for the $n$-th product. Let $P_{n}^{k-1}=\left[p(n)_{i, j}^{k-1}\right]$ be the matrix of the flow capacity for the $n$-th product. We can determine the flow capacity of the $j$-th production stand in the $i$-th logistic block for the $n$-th element of the order vector $Z$ in the form (1).

$$
p(n)_{i, j}^{k-1}=g_{n, j}-s(n)_{i, j}^{k-1}
$$

The matrix $T^{p r}=\left[\tau_{n, i}^{p r}\right]$ includes production times for the $n$-th product in the $j$-th production stand of the $i$-th logistic block. The vector $T^{r e p l}=\left[\begin{array}{lllll}\tau_{1}^{r e p l} & \ldots & \tau_{i}^{r e p l} & \ldots & \tau_{I}^{r e p l}\end{array}\right]$ includes replacement times for the tools in the logistic system. If $\tau_{n, i}^{p r} \leq \tau_{n, i+1}^{p r}$, then the $j$-th production stand of the $i$-th logistic block becomes blocked while manufacturing the $n$-th product so there is the need to activate the production stand $m_{i+1, j+1}$. Let $V=\left[v_{n, i}\right]$ be the production rate vector where $v_{n, i}$ is the number of units of the $n$-th product made in the time unit. The total manufacturing time of all elements from vector $Z$ is given by 
(2), where $y_{i}^{k}=1$ if the replacement procedure of the tool in the $i$-th stand is carried out. Otherwise, $y_{i}^{k}=0$

$$
T=\sum_{n=1}^{N} \sum_{i}^{I} \tau_{n, i}^{p r}+\sum_{k=0}^{K} \sum_{i}^{I} y_{i}^{k} \tau_{i}^{r e p l}
$$

\section{THE MANUFACTURING CRITERION}

The lost flow capacity criterion takes the form (3) and is accompanied by the tool replacement bound in the form (4) as well as by the order bound in the form (5) where $p_{n, i}^{k}$ is the lost flow capacity of the $j$-th stand of the $i$-th logistic block at the $k$ th stage, $c$ is the maximal allowable tool replacement time, $x_{n}^{k}$ is the number of units of the $n$-th element realized at the $k$-th stage, $\tau_{i}^{\text {repl }}$ is the replacement time of the used tool in the $j$-th stand of the $i$-th logistic block. The lost flow capacity criterion is reduced to the replacement time of tools and order bounds.

$$
\begin{gathered}
Q_{2}=\sum_{k=1}^{K} q_{2}^{k}=\sum_{k=1}^{K} \sum_{i=1}^{I} y_{i}^{k} \sum_{j=1}^{I} p_{n, i}^{k} \rightarrow \min \\
\sum_{i=1}^{I} y_{i}^{k} \tau_{i}^{r e p l} \leq c \\
\sum_{n=1}^{N} x_{n}^{k} \leq z_{n}
\end{gathered}
$$

\section{EQUATIONS OF STATE}

The state of the logistic system changes according to the form (6).

$$
S(n)^{0} \rightarrow S(n)^{1} \rightarrow \ldots \rightarrow S(n)^{k-1} \rightarrow \ldots \rightarrow S(n)^{K}
$$

The state of the $j$-th production stand of the $i$-th logistic block changes consequently according to the form (7), which is written as $s(n)_{i, j}^{k}=s(n)_{i, j}^{k-1}+x_{n}^{k}$, otherwise $s(n)_{i, j}^{k}=s(n)_{i, j}^{k-1}$.

$$
s(n)_{i, j}^{0} \rightarrow s(n)_{i, j}^{1} \rightarrow \ldots \rightarrow s(n)_{i, j}^{k} \rightarrow \ldots \rightarrow s(n)_{i, j}^{K}
$$

Let $\rho_{i}$ be the tool to be replaced, $1 \leq \rho_{i} \leq I$. The state of the $j$ th production stand of the $i$-th logistic block in case of replacement of tools changes according to the specification (8).

$$
s(n)_{i, j}^{k}= \begin{cases}s(n)_{i, j}^{k-1} & \text { if } i \neq \rho \text { at the } k \text {-1 stage } \\ 0 & \text { otherwise, }\end{cases}
$$

The order vector is modified after every decision about production according to (9).

$$
z_{n}^{k}= \begin{cases}z_{n}^{k-1}-x_{n}^{k} & \begin{array}{l}
\text { if the number of units } x_{n}^{k} \\
\text { of the } n \text {th orderis realized } \\
\text { at the } k \text { stage }
\end{array} \\
z_{n}^{k-1} & \text { otherwise }\end{cases}
$$

\section{THE HEURISTIC ALGORITHMS}

The heuristic algorithm determines the order element characterized by the maximal relative order coefficient $\gamma_{n}^{k-1}$ in the state $S(n)^{k-1}$.
To produce the element $a$, the condition in the form (10) must be met where $\gamma_{n}^{k-1}=\frac{z_{n}^{k-1}}{z_{n}^{0}}$. It is assumed that the orders are realized one after another that is to say each order element $z_{n}$ in the state $S_{n}^{k-1}$ is reduced partly

$$
\left(q^{k}=a\right) \Leftrightarrow\left[\gamma_{a}^{k-1}=\max _{1 \leq n \leq N} \gamma_{n}^{k-1}\right]
$$

\section{CONCLUSIONS}

The problem highlighted in this paper discusses the issue of the sample serial production system with no buffer stores. Production stands are arranged in series. Each stand can also be multiplied by extra parallel stands of exactly the same production possibilities. The system delivers ready products corresponding to the elements of the order vector. The main goal is to fulfil the task set by the criterion. The heuristic algorithm proposed in the paper enables the operator to choose the satisfactory production sequence on the basis of which a certain element of the order vector is determined for realization. The use of one specified algorithm does not mean that we will achieve the result satisfying the given criterion. It is advisable to implement other algorithms and decide which one minimizes the order realization time, the loss of residual capacity and the total replacement time or satisfies a hypothetical customer's demand not specified hereby. To achieve a satisfactory result a big number of simulations must be carried out. Assuming that the results of calculations which are made for one chosen heuristic algorithm do not deliver a satisfactory solution, there arises a need to test other algorithms.

\section{ACKNOWLEDGEMENTS}

This work was supported by the Ministry of Education of the Czech Republic under grant No. MEB 051024 and No. MEB051031.

\section{REFERENCES}

Bucki, R. \& Chramcov, B. (2011). Control of the serial production system. In: Recent Researches in Automatic Control, Proceedings of 13th WSEAS Int. Conference on Automatic Control, Modelling \& Simulation. Lanzarote, May 2005, pp. 352-356, ISBN 978-1-61804-004-6, WSEAS Press

Chramcov, B., Beran, P., Daníček, L. \& Jašek, R. (2011). A simulation approach to achieving more efficient production systems, International Journal of Mathematics and Computers in Simulation [online], Volume 5, Issue 4, June 2011, [cit. 2011-06-30]. Available from: http://www.naun.org/journals/mcs/, ISSN 1998-0159

Michalewicz, Z. \& Fogel, D. B. (2004). How to Solve It: Modern Heuristics, Springer, 2nd ed. Revised and Extended, 554 p. ISBN 978-3-540-22494-5

Modrák, V. \& Pandian, R.S. (2010). Flow shop scheduling algorithm to minimize completion time for $\mathrm{n}$-jobs $\mathrm{m}$ machines problem. Tehnicki Vjesnik, 17 (3), 2010, pp. 273 278. ISSN 1330-3651

Modrák, V. (2009). Case on manufacturing cell formation using production flow analysis, Proceedings of World Academy of Science, Engineering and Technology, Volume 37, 2009, pp. 519-523. ISSN: 2010376X

Schmidt, R. (1988). Application of simulation for order sequencing in flow-shop production systems. In Proceedings of the 21st annual symposium on Simulation (ANSS '88), Los Alamitos, CA, USA, pp. 71-89, IEEE Computer Society Press 